\title{
Super-resolved 3D mapping of molecular orientation with vibrational techniques
}

\author{
Paulina Koziol $^{1,2}$, Karolina Kosowska ${ }^{1}$, Danuta Liberda ${ }^{1}$, Ferenc Borondics ${ }^{3}$, Tomasz P. Wrobel ${ }^{1 *}$ \\ ${ }^{1}$ Solaris National Synchrotron Radiation Centre, Jagiellonian University, Czerwone Maki 98, 30-392 \\ Krakow, Poland. \\ ${ }^{2}$ Institute of Physics, Jagiellonian University, Lojasiewicza 11, 30-348 Krakow, Poland \\ ${ }^{3}$ Synchrotron Soleil, L'Orme des Merisiers, Saint-Aubin - BP 48, 91192, Gif-sur-Yvette, France \\ *email: tomek.wrobel@uj.edu.pl
}

\begin{abstract}
When a sample has an anisotropic structure, it is possible to obtain different information, when changing polarization of incident light. Using polarized light of a single vibrational band to determine the in-plane orientation and internal ordering of a sample is a typical practice in materials science. Acquiring mapping data at four different polarizations with a stationary sample than just at two polarizations offers much more insight into the sample structure with proper mathematical treatment. A concurrent analysis of two vibrational bands with perpendicular transition moment orientations allows the understanding of the orientational ordering in three dimensions. We show here, to the best of our knowledge, the first application of concurrent analysis to IR spectromicroscopy data and obtain orientation angles of a model spherulite polycaprolactone sample. Moreover, we show that this method can be easily applied to high resolution, diffraction limited FT-IR and Raman imaging and even to sub-diffraction limit O-PTIR imaging. Due to the non-tomographic experimental approach, no image distortion is visible and nanometer scale orientation domains can be observed. 3D bond orientation maps will enable in depth characterization of sample structure in a quantitative manner enabling more precise control of their physicochemical properties and function.
\end{abstract}

\section{Keywords}

3D molecular orientation, Linear Polarization, FT-IR Imaging, O-PTIR, Raman, Polycaprolactone, Spherulite

\section{Introduction}

Chemical characterization and visualization of nanoscale structures and interphases in materials like semi-crystalline polymers (spherulites, nanofibers), nanocomposites and biological systems (proteins, peptides) are critical for deeper understanding of the correlation between "superstructure" of material and its physicochemical and biological properties. Reorganization at the nano-structural level makes it even possible to obtain metal-like thermal conductors from polymers, commonly known as insulators. $\mathrm{Hu}$ et al. presented polyethylene film with parallel aligned nanofibers with thermal conductivity two orders-of-magnitude greater than normal polymer [1]. Research is also ongoing into flexible energy stores based on polymer nanohydrogels [2], e-skin for medicine [3] and intelligent (biomimetic) scaffolds to induce a cellular response [4]. Several methods can be used to evaluate the structural and compositional properties of nanomaterials like nanoparticles and nanofibers. Techniques such as X-ray Diffraction probe only crystalline phases with enough symmetry, while Polarization ModulationInfrared Reflection-Absorption Spectroscopy (PM-IRRAS), Calorimetry or Nuclear Magnetic Resonance provide only bulk information (or very limited spatial resolution). Electron Microscopy (EM) 
and Atomic Force Microscopy (AFM) offer fantastic spatial resolution at the cost of specificity and sample preparation requirements. The family of optical techniques (fluorescence, pump-probe, Raman and infrared) seems to be the middle-ground, providing a wealth of information while being spatially diffraction limited in the $200 \mathrm{~nm}$ up resolution range [5], however, not offering fully 3D resolved information apart for single analytes.

Fourier-transform infrared spectroscopy (FT-IR) in combination with chemometrics is an intensively developed, powerful tool for investigation of material's chemical structure with simultaneous microscopic visualization [6]. This nondestructive, information rich and label-free technique has been successfully applied for years in material science. One of the shortcomings of the FT-IR imaging method is the inherent low spatial resolution. In any type of (far field) optical microscopy the spatial resolution is restricted by the diffraction limit which is in the order of the wavelength $\lambda$ of the light. For midinfrared spectroscopy the maximum spatial resolution is $\sim 2-10 \mu \mathrm{m}$. An emerging technique that overcomes this limitation by detecting photothermal expansion caused by IR absorption using an optical probe (a visible laser) has recently been developed under the name of Optical Photothermal Infrared spectroscopy (O-PTIR) [7]. This far-field microscopic technique overcomes several limitations of traditional IR imaging: sample thickness, transmissive substrate, or presence of water, while offering imaging with an effective $400 \mathrm{~nm}$ spatial resolution [8].

IR imaging with linear polarization can provide new and useful information about chemically specific orientation of macromolecules. When a sample has anisotropic structure, changing the polarization of incident light in a microscopic investigation can reveal additional information. The in-plane direction of transition moments of vibrational bands in the sample can be described by azimuthal angle $(\psi)$, when a linear polarization-based method is applied. The IR two-polarization method was used to examine orientation in polymeric samples [9] and the Raman counterpart has been used for years [10]. Next, the four-polarization method (4P) has been proposed, which enabled study of the three-dimensional organization of macromolecules in complex biological systems. The first applications of IR imaging with $4 \mathrm{P}$ on polymeric systems [11]-[15] were recently followed by human tissue microarrays analysis by Koziol et al. in 2020 [16].

Until now, experiments focused on using a single vibrational band to retrieve the in-plane orientation and internal ordering of samples. $4 \mathrm{P}$ is experimentally a simple (no sample tilting) framework that offers much more with proper mathematical treatment. A concurrent analysis of two vibrational bands of roughly perpendicular transition moment orientations was suggested and results in full 3D angles (inplane and out-of-plane) retrieval. We show here, to the best of our knowledge, the first application of concurrent analysis to IR imaging data and obtain orientation angles of a model spherulite polycaprolactone sample. Moreover, we show that this method can be applied to diffraction limited FTIR and Raman imaging and even to sub-diffraction limit O-PTIR imaging. Due to the stationary experimental approach, no image distortion is visible and nanometer scale domains can be observed.

\section{Materials and methods}

\section{Spherulite sample preparation}

Polycaprolactone (PCL) of $\mathrm{M}_{\mathrm{n}}=80000 \mathrm{~g} / \mathrm{mol}$ was purchased from Sigma Aldrich Sp. z o.o (Poznan, Poland). Chloroform for analysis (99.8\%), amylene stabilized was obtained from Idalia Sp.J (Radom, Poland). PCL has a melting point $\left(\mathrm{T}_{\mathrm{m}}\right)$ of $60{ }^{\circ} \mathrm{C}$ and glass transition temperature $\left(\mathrm{T}_{\mathrm{g}}\right)$ of around $-60{ }^{\circ} \mathrm{C}$. PCL film was prepared from solution (1.2 $\mathrm{g}$ of granular polymer in $80 \mathrm{~mL}$ of chloroform). The system was left on a magnetic stirrer for $24 \mathrm{~h}$ at room temperature to allow complete dissolution. The PCL film of thickness around $10 \mu \mathrm{m}$ was prepared by casting solution onto calcium fluoride $\left(\mathrm{CaF}_{2}\right)$ slide. It was 
placed in a fume hood for evaporation of the solvent per night. Finally, slide with PCL film was heated up from room temperature to $75^{\circ} \mathrm{C}$ on a heating plate and left for $1 \mathrm{~h}$. The film was melted to erase the polymer structure. Next, temperature was lowered to $35^{\circ} \mathrm{C}$. After $5 \mathrm{~h}$ of the PCL film crystallization at a constant temperature, it was cooled naturally to the room temperature.

\section{FT-IR imaging}

FT-IR measurements were done with Bruker Vertex70v Spectrometer coupled with Hyperion 3000 Microscope equipped with a 64x64 FPA MCT detector. A 15x Cassegrain objective (NA=0.4) was used giving $2.7 \mu \mathrm{m} \times 2.7 \mu \mathrm{m}$ projected pixel size. To perform measurements with linearly polarized light, two wire grid polarizers were inserted before the objective and at the bottom of the condenser. Simultaneous rotation of both polarizers aligned in parallel allowed achieving desired polarizations four datasets were collected, with polarizations corresponding to $0^{\circ}, 45^{\circ}, 90^{\circ}$ and $135^{\circ}$. To provide pixelto-pixel spatial alignment of all datasets, a motorized sample stage was programmed to repeat the same positions (for each polarization). Data acquisition was done in $3850 \mathrm{~cm}^{-1}$ to $900 \mathrm{~cm}^{-1}$ spectral range, with $8 \mathrm{~cm}^{-1}$ spectral resolution and zero filling factor of 1 . Sample and background spectra were coaveraged 4 and 64 times, respectively.

\section{O-PTIR imaging}

O-PTIR imaging was done using a mIRage O-PTIR microscope. Quantum cascade laser (QCL) was used as a pump beam with a pulse rate of $100 \mathrm{kHz}$ in a $2.4 \%$ duty cycle. Images were collected for five wavenumbers: 1736, 1368, 1296, 1246, $1170 \mathrm{~cm}^{-1}$ with corresponding QCL laser powers: 4, 24, 24, 18 , $18 \%$, respectively. $532 \mathrm{~nm}$ laser with $0.5 \%$ power was used as a probe beam. Measurements were performed in reflection mode with an avalanche photodiode detector (APD) and $200 \mathrm{~nm}$ step. The background was measured once, on the sample-free region of optical slide. Since QCL laser is intrinsically linearly polarized, to obtain different polarizations sample was rotated by roughly 45 degrees clockwise. This procedure was done three times to obtain four polarization datasets at $0,45,90$, and 135 degrees. The measurement region was adjusted each time the sample was rotated, to cover whole spherulite of interest.

\section{Raman imaging}

Raman measurements of PCL film were performed using a WITec confocal CRM alpha 300 Raman microscope with 50x objective. The spectrometer was equipped with an air-cooled solid-state laser operating at $532 \mathrm{~nm}$ and a CCD detector. The laser power measured before the objective was approximately $50 \mathrm{~mW}$. The spectra were recorded with spectral resolution of $4 \mathrm{~cm}^{-1}$ and an integration time of $0.1 \mathrm{~s}$. The single half-wave plate was placed above objective to control laser polarization direction. Four polarizations of interest $\left(-30^{\circ}, 0^{\circ}, 60^{\circ}\right.$ and $\left.120^{\circ}\right)$ were achieved by the rotation of wave plate. A rectangular fragment of spherulite from borderline of the neighboring spherulite to the nucleation site was measured with $200 \mathrm{~nm}$ step (size: 150x450 steps).

\section{Pre-processing}

\section{FT-IR data}

Spectral and spatial data quality was increased by the application of Minimum Noise Fraction (MNF) denoising, with 15 bands used for reconstructions [17], [18]. Furthermore, a local linear baseline was applied to the spectral ranges corresponding to vibrations of crucial chemical bonds, which allowed mitigation of scattering artifacts. Single intensities (within corrected ranges) were used for transition 
dipole moment orientation calculations, with the attention focused on 1720, 1365, 1292, 1238 and 1165 $\mathrm{cm}^{-1}$ set, complementary to collected O-PTIR data.

\section{O-PTIR data}

Due to the fact, that during O-PTIR imaging different linear polarizations were achieved by sample rotation (not by polarization control), an additional preprocessing step was required to spatially align four datasets. Final sample orientation was defined by $90^{\circ}$ polarization dataset (closest to FT-IR results orientation), whereas $0^{\circ}, 45^{\circ}$ and $135^{\circ}$, datasets were warped by global rotation and translation of each image. Calculation of rotation angles $\left(85.7^{\circ}, 136.9^{\circ}\right.$ and $\left.42.5^{\circ}\right)$ used for images transformation was done using line marked on the sample before measurements. Following, images were translated referring to characteristic sample structures - spherulite edges. During data manipulation we noticed that intensities for the last polarization dataset $\left(135^{\circ}\right)$ were significantly higher and therefore not comparable to other polarizations. To overcome this artifact (most probably coming from QCL laser power), all images collected with O-PTIR technique were divided by its median value. Finally, aiming to prepare data for further absorptance calculations in 3D orientation method, intensities for each spectral variable (cumulative data for all four polarizations) were rescaled to span within intensities range corresponding to absorption values exhibited by FT-IR data. Furthermore, additional datasets were generated by binning $13 \times 13$ pixels (giving $2.6 \mu \mathrm{m}$ pixel), with the aim to later compare O-PTIR and FTIR data (2.7 $\mu \mathrm{m}$ pixel).

\section{Raman data}

The data treatment started with cosmic spike removal and Raman shift calibration to Si bands. This was followed by denoising with Principal Component Analysis (PCA), with 15 Principal Components used for reconstructions, which significantly increased spectral and spatial data quality. One of the polarization datasets exhibited slightly higher global intensities (distorting orientation calculations), therefore, all images were divided by its median value. Global baseline correction was done using the rubber-bands method with 13 spectral ranges used for linear corrections. Similarly to O-PTIR, Raman spectra also required intensities rescaling for further absorptance calculations. However, due the lack of some bands present in FT-IR spectra, data was scaled so that intensities for the most intensive band in Raman spectra are similar to absorbance of most intensive bands in FT-IR data.

\section{Theoretical approach}

Anisotropic materials, such as fibers or polymers with long molecular chains exhibit high absorption dependence from polarization direction of the incoming light, both in IR spectroscopy and other spectroscopic techniques. This effect, called linear dichroism is extensively applied in molecular orientation studies. In polarized infrared spectroscopy (FT-IR and O-PTIR), absorption by specific molecular vibration is highest when the transition dipole moment $\frac{\partial \mu}{\partial Q}$ vector is aligned with the polarization [19]. Schematic example of FT-IR instrumentation setup is shown in Figure 1a. Similarly, in Polarized Raman spectroscopy scattering reaches a maximum for best alignment of polarization with highest polarizability tensor change $\frac{\partial \alpha}{\partial Q}$, resulting in maximum band intensity observed in Raman spectrum [20]. Hence, analysis of spectra collected for different incident light polarizations can lead to conclusions about chemical bonds and molecular orientation, with dichroic ratio $(D)$ being one of the most widely applied parameters. $D$ is defined as ratio of absorbance (or intensity) for parallelly and perpendicularly polarized light (with respect to molecular axis). Most of the studies carried out within this area used two perpendicular polarizations, which required specific sample placement - analyzed 
molecules must have been parallel to one polarization and perpendicular to another. In other cases, $D$ would exhibit misleading values. Such constraint makes this method appropriate only for simple systems with molecules aligned alongside each other, excluding systems with molecules changing directions throughout the sample.

\section{Four-polarization method in $2 D$ orientation}

The disadvantages of two-polarization method have been aided by development of four-polarization approach which allows molecular bond orientation determination, independently from the choice of incident light polarization. Absorbance dependence from linear polarization may be describes by a simple formula, as follows [11]-[13]:

$$
A_{\eta}=\frac{A_{\max }+A_{\min }}{2}+\frac{A_{\max }-A_{\min }}{2} \cos 2(\eta-\psi)
$$

where $A_{\min }$ and $A_{\max }$ are minimum and maximum absorbance, $\eta$ is an incident polarization direction and $\psi$ is a polarization direction when $A_{\eta}=A_{\max }$. Collecting data with four different linear polarizations (for each pixel) gives possibility to experimentally find the shape of $A_{\eta}$, as shown in Figure 1b. The applied here mathematical approach was based on non-linear fit of cosine function and extraction of $A_{\min }, A_{\max }$ and $\psi$ parameters. Considering dipole moment $\mu$ being oriented in 3D space, $\psi$ gives limited information about in-plane orientation of this vector. Exemplary results visualization (Figure 1d) clearly shows the big disadvantage of 2D method, where extracted information fully omits vertical component of orientation. Based on $A_{\min }$ and $A_{\max }$, one can also compute Herman's in-plane orientation function defined as [11]-[13]:

$$
f=\frac{D-1}{D+2} \frac{2}{3 \cos ^{2} \kappa-1}
$$

where $D=A_{\max } / A_{\min }$ and $\kappa$ describes angle between main molecular axis and transition dipole moment. Herman's function is equal to the second order coefficient of series of Lagrange polynomials, approximating the orientation distribution function (ODF) of a molecule with cylindrical symmetry [21], [22]. Therefore, it might be used to describe level of molecular orientation of the sample. Regardless of this example being described for FT-IR data characterized by absorbance values - the same approach is also suitable for Raman scattering intensity.

\section{Four-polarization in $3 D$ orientation}

Vertical orientation issues, mentioned in above section, were recently approached be Lee, who proposed a mathematical method allowing determination of 3D angles and order parameter for molecular orientation imaging with FT-IR [23]. Derivation is established on the grounds of classic electromagnetic wave theories and uses two non-parallel transition dipole moments simultaneously: primary $\left(\mu_{1}\right)$ associated with the main molecular axis (for example main molecular chain vibration) and secondary $\left(\mu_{2}\right)$ representing an orthogonal vibration. Based on that, absorptance $(\alpha=1-T)$ dependence from polarization direction $\eta$ for vectors $\mu_{1}$ and $\mu_{2}$ is described as [23]:

$$
\begin{gathered}
\frac{\alpha_{1}(\eta)}{\alpha_{1}^{o}}=\left\langle P_{2}\right\rangle \sin ^{2} \theta \cos ^{2}(\eta-\psi)+\frac{1}{3}\left(1-\left\langle P_{2}\right\rangle\right), \\
\frac{\alpha_{2}(\eta)}{\alpha_{2}^{o}}=\left\langle P_{2}\right\rangle[\cos \theta \cos \phi \cos (\eta-\psi)+\sin \phi \sin (\eta-\psi)]^{2}+\frac{1}{3}\left(1-\left\langle P_{2}\right\rangle\right),
\end{gathered}
$$


where $\psi, \theta$ and $\phi$ are angles describing 3D orientation of $\mu_{1}$ and $\mu_{2}$ vectors (shown in Figure 1c), $\left\langle P_{2}\right\rangle$ is the order parameter, whereas $\alpha^{\circ}$ contains only non-orientational quantities. Similarly like in 2D approach, non-linear functions were fitted to data points from four polarization datasets, which allowed reconstruction of experimental shape of absorptance dependence from incident polarization. As presented in Figure 1c, following parameters pairs were later extracted from obtained experimental functions: $\alpha_{1, \max }, \eta_{1, \max }, \alpha_{1, \min }, \eta_{1, \min }, \alpha_{2, \max }, \eta_{2, \max }$ and $\alpha_{2, \min }, \eta_{2, \min }$. Those parameters were necessary to further calculate intermediate parameters, defined as [23]:

$$
\begin{gathered}
M \equiv\left(\frac{\alpha_{1, \max }}{\alpha_{1, \min }}-1\right)=\frac{3\left\langle P_{2}\right\rangle}{1-\left\langle P_{2}\right\rangle} \sin ^{2} \theta \\
N \equiv\left(\frac{\alpha_{2, \max }}{\alpha_{2, \min }}-1\right)=\frac{3\left\langle P_{2}\right\rangle}{1-\left\langle P_{2}\right\rangle}\left(\cos ^{2} \phi \cos ^{2} \theta+\sin ^{2} \phi\right) \\
\Delta \eta=\eta_{2, \max }-\eta_{1, \max } \\
P=\frac{1}{2}\left[(M+N)+\sqrt{(M-N)^{2}+4 M N \cos ^{2} \theta \Delta \eta}\right]
\end{gathered}
$$

One may notice that for $\frac{\alpha_{1 \max }}{\alpha_{1, \min }}$ and $\frac{\alpha_{2, \max }}{\alpha_{2, \min }}$ ratios observed in definition of $M$ and $N, \alpha^{\circ}$ may be considered as a constant, thus, have no impact in further calculations. Having intermediate parameters and after equations manipulation, it is a straight way to find results for $\mu_{1}$ and $\mu_{2}$ orientations along with order parameter [23]:

$$
\begin{gathered}
\left\langle P_{2}\right\rangle=\frac{P}{P+3}, \\
\psi=\eta_{1, \text { max }} \\
\theta=\sin ^{-1} \sqrt{\frac{M}{P}} \\
\phi=\cos ^{-1}\left[ \pm \sqrt{\left.\frac{(P-N)}{M}\right] .}\right.
\end{gathered}
$$

The order parameter $\left\langle P_{2}\right\rangle$, which is equal to second-order coefficient of an ODF of molecule with cylindrical symmetry, is used as a quantity describing the breadth of ODF [23]. An example of 3D orientation results is shown in Figure 1e. It should be noted that all vectors have the same length and only vectors directions are of significance. Since sections $d$ and e of Figure 1 show results of corresponding sample regions, it is clearly visible how important advantage one gains by using method for 3D orientation determination. Even though this method was determined for FT-IR method, the adaptation for O-PTIR and Raman spectra was done by transferring intensities to reach values corresponding to FT-IR absorbance, as it was briefly described in pre-processing section.

\section{Results}

Figure 1 presents conceptually the four-polarization method supplemented by concurrent vibration analysis leading to retrieval of 3D angles. Four different linear polarization angles allow to probe a sinusoidal function that comes from orientation dependent absorption. Analysis of a single vibration 
leads to obtaining 2D in-plane angle of orientation, while simultaneous analysis of two near-orthogonal vibrations retrieves full 3D angles.

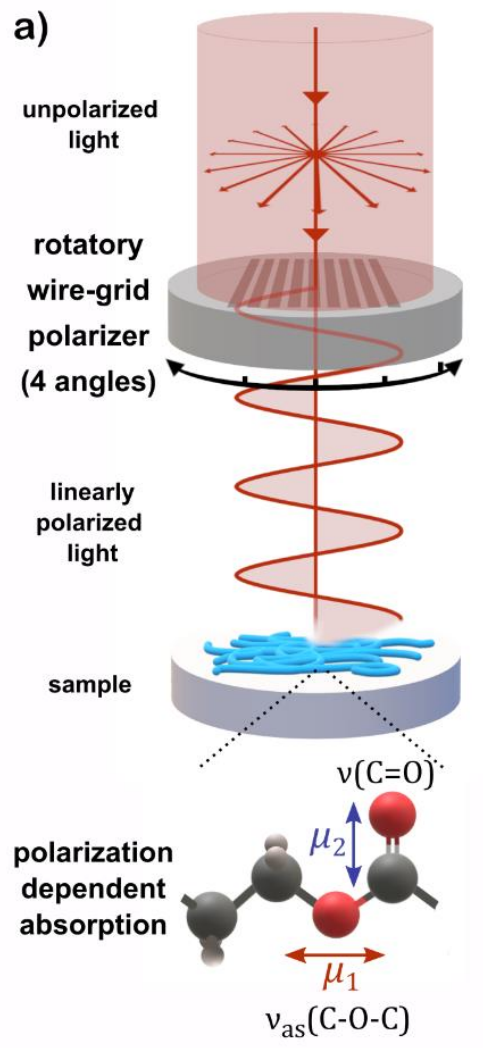

d)

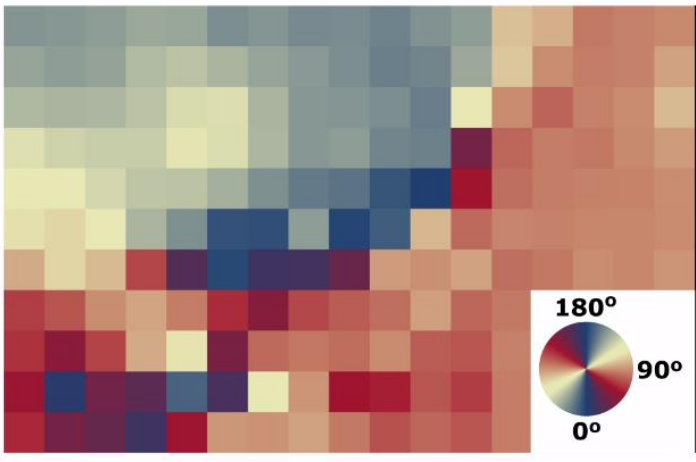

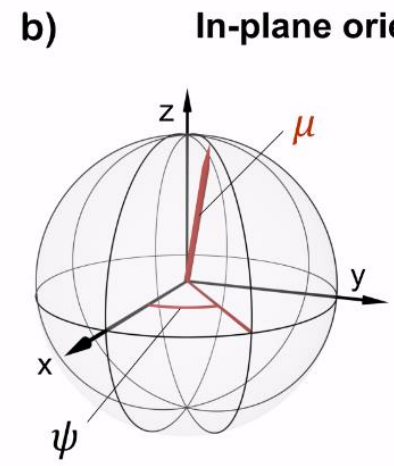

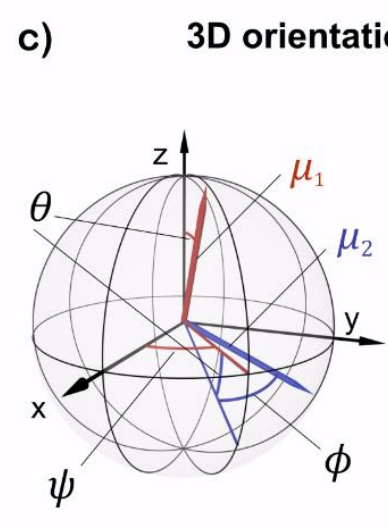

e)

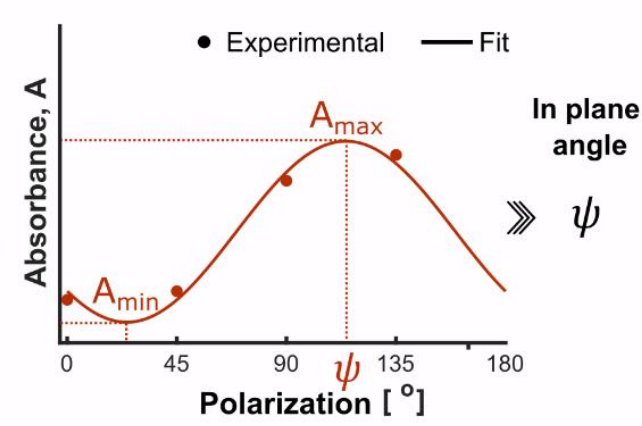

ion - two vibrations $\left(\mu_{1}\right.$ and $\left.\mu_{2}\right)$

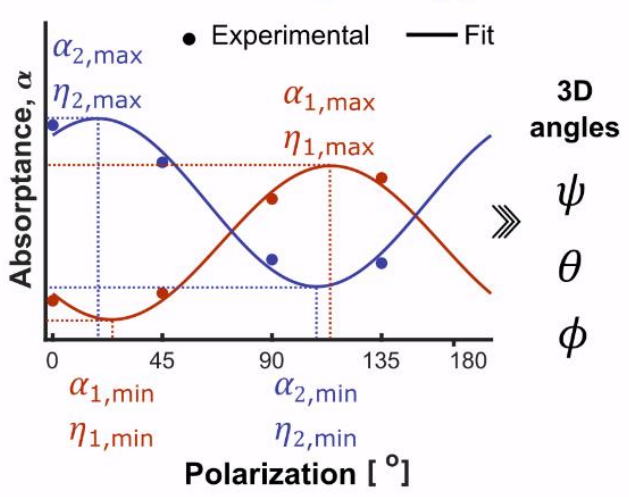

3D orientation

Figure 1. a) Schematic drawing of Polarized FT-IR experimental setup including absorbance dependence of molecule from orientation of incident polarization. b) Visualization of approach for four-polarization method used to determine in-plane orientation (2D) of transition dipole moment $\mu$ oriented in space. c) Visualization of approach for four-polarization method used to determine 3D orientations of transition dipole moments $\mu_{1}$ and $\mu_{2}$. d), e) Comparison of results visualization for 2D and $3 \mathrm{D}$ methods, for the same sample region.

To present the capabilities of this approach a model polymer was selected and measured using FT-IR, O-PTIR and Raman mapping. Poly- $\varepsilon$-caprolactone (PCL) is often used as model system for polymers crystallization studies due to its simple chemical and crystal structure, similar to the one exhibited by 
polyethylene (PE). However, it should be noted that carbonyl groups of PCL result in a slightly twisted chain formation of the unit cell in comparison to PE. PCL forms orthorhombic crystal structure with planar, zig-zag chain conformation [24]. In left panel of Figure 2a the unit cell of PCL is presented. Undercooling solution or melt of semi-crystalline polymer often crystalizes to the spherulites form and the crystallization of each one start at the nucleation site. Spherulite can be described as spherically symmetrical clusters of crystals. However, in the most cases of polymer films, spherulites appear in the flat form with radial symmetry and grow until impact stops the process (Figure 2b). Long fibrils which are clearly visible under optical microscope, are made of lamellas oriented in parallel and separated by amorphous regions. The free space between fibrils can be filled with amorphous phase or stay empty. To uniformly fill the space, new fibrils must be generated during growth. Processes like heterogenous nucleation on already existing fibril and branching generate new, neighboring fibrils (right panel of Figure 2a). Several studies with AFM microscopy showed that in the early stages of growth, spherulites appear in the form of sheaves of lamellae. During growth crystals splay apart from each other and fill the space [25]. The single lamella is made of highly oriented chains folded back several times, so the single chain passes through the lamellar structure more than once. This is presented in the frontal view of single lamellae section in Figure 2a. The amorphous regions between lamellae are created of folds, chain ends and polymer chains without order. The chains which connect neighboring lamellas are described as "tie chains". Lamellas grow along crystallographic axis b and they can exhibit different types of orientation on substrate, which can change from "flat-on" to "edge-on" as it is shown in the middle part of Figure 2a. Spherulites termed as banded consist of twisted lamellar crystals. The unbalanced surface stresses are the driving forces of lamellar [18]. 
a)

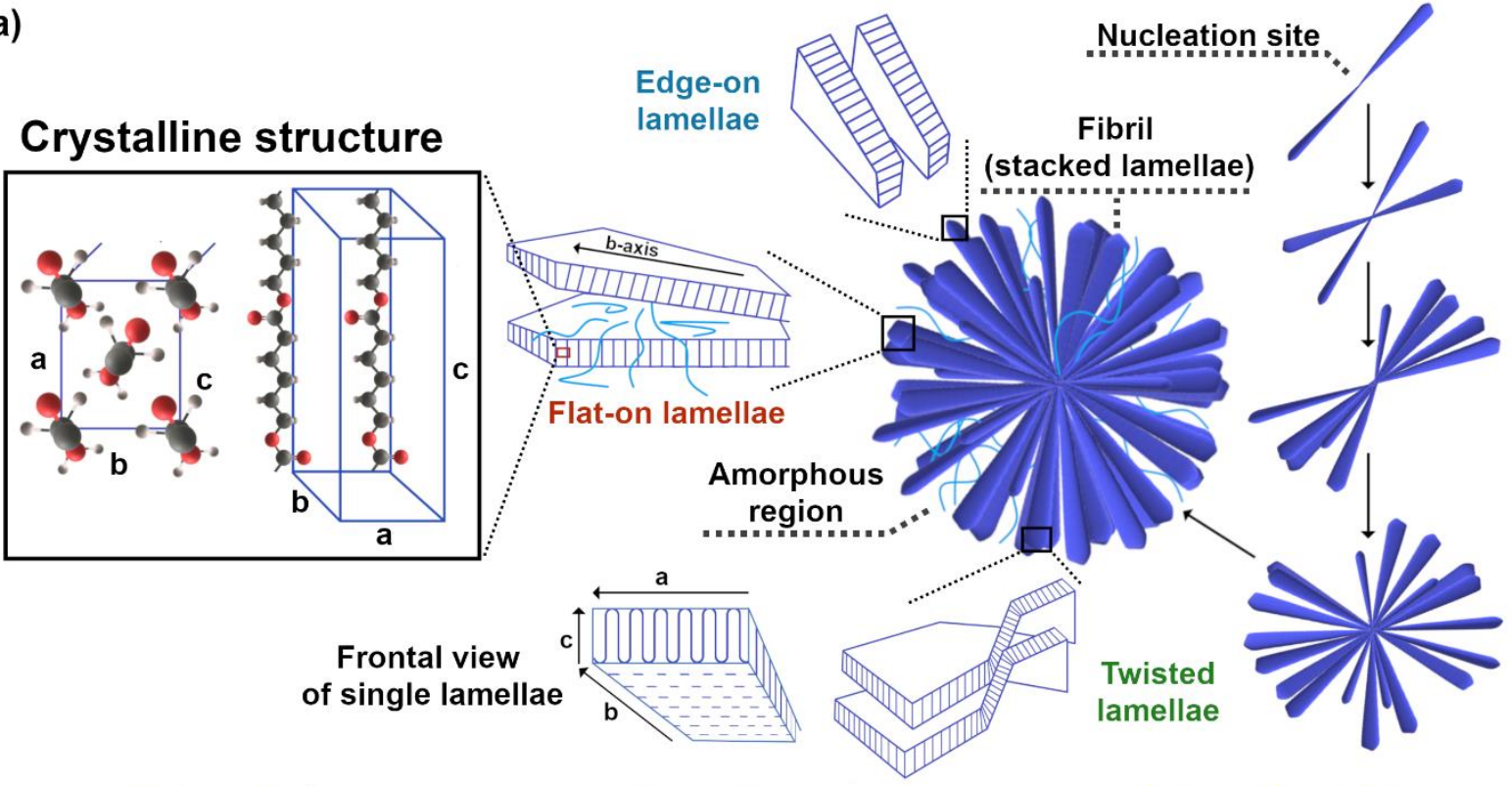

Unit cell: Å

Lamellae: $\mu \mathrm{m}$

Spherulite: $100 \mu \mathrm{m}$

b) Polymer film
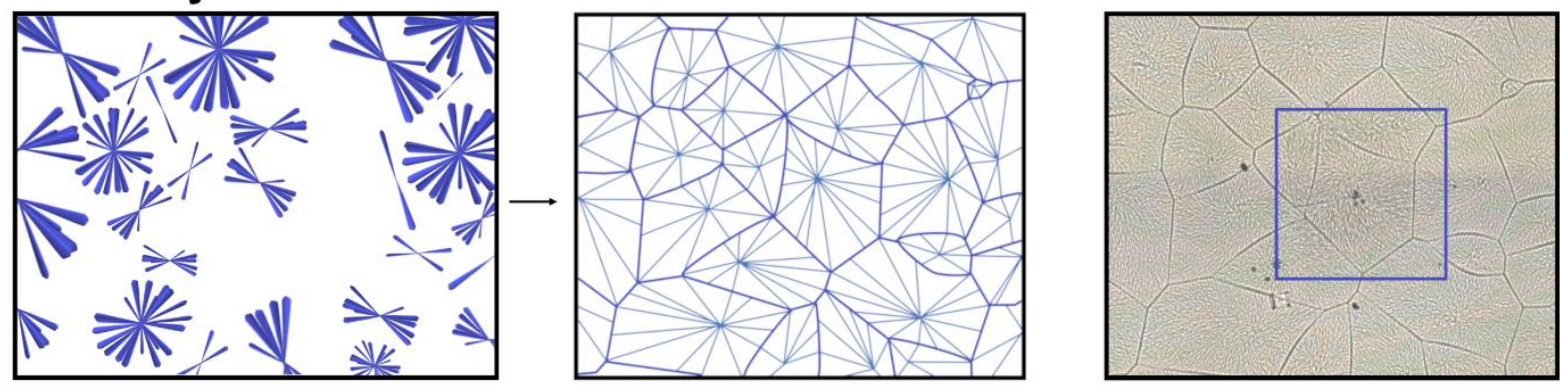

Figure 2. Schematic representation of the hierarchical structure of the polymer spherulite. In panel (a) the orientation of polymer chains and crystalline unit cell of PCL is indicated together with the crystal axes of the lamella made of tightly folded chains. Spherulite is composed of highly ordered lamellas,

connected by amorphous region, in different orientation: the "edge-on" orientation, "flat-on"

orientation and twisted form. Spherulite growth during polymer crystallization starts in nucleation site. The size is controlled by the nucleation of spherulite and is in the tens of $\mu \mathrm{m}$ range with an approx. 10 $\mu \mathrm{m}$ thickness. Panel (b) shows the schematic illustration of polymer film crystallization and optical microscopic image of PCL film with marked spherulite selected for detailed research. With large number of nucleation sites, spherulites interact with each other upon growth.

Data for polycaprolactone spherulite sample described above and characterized in Figure 2 were collected using three spectroscopic techniques: FT-IR, O-PTIR and Raman spectroscopy, all at their diffraction limit. Each technique possesses individual features highly determining measurement time, e.g., detector size or measurement step, integration time, objective, etc. Furthermore, measurement time is strongly increased (in comparison with experiments done with unpolarized light) by the requirement of four polarization datasets collection. Hence, FT-IR with matrix detector and lowest spatial resolution allowed measurements of larger area of polymer film than other modalities, offering at the same time full spectral coverage. O-PTIR on the other hand provides significantly higher spatial detail $(200 \mathrm{~nm}$ pixel size), but to cover region of full spherulite of interest, only 5 bands were chosen for measurements (discrete frequencies). Therefore, from the whole PCL film, single spherulite was selected for detailed analysis, with its shape and size presented in Figure 3a. Optical image of this exact spherulite was also 
shown in Figure 2b. Raman measurements were narrowed to a smaller spherulite region, but still covering nucleation site and spherulite edge. Keeping measurement step of $200 \mathrm{~nm}$ allows to directly compare Raman and O-PTIR results in terms of spatial detail. Comparison of those matching regions is shown in Figure 3c, whereas their placement within the sample is marked with blue rectangle in Figure 2a. To visualize results, including sample heterogeneity, images of intensities ratios for two perpendicular polarizations are presented in Figure 3a,c. Specific bands were selected for each method, $v_{\text {as }}(\mathrm{C}-\mathrm{O}-\mathrm{C})$ for FT-IR and O-PTIR and $\omega\left(\mathrm{CH}_{2}\right)$ for Raman - both with transition moment changes approximately parallel to main molecular axis. It is clearly visible that there is strong absorption dependence from incident polarization direction - some regions are highlighted while others are clearly dimmed, resulting in a typical pattern. Different spherulites might be recognized based on FT-IR intensity image of PCL film. Moreover, nucleation site is nicely visible for all techniques. Exemplary spectra (unpolarized) from PCL spherulite sample are presented for each technique in Figure 3b. Nice correspondence between FT-IR and O-PTIR techniques is observed. Moreover, as expected based on selection rules, some bands clearly visible in infrared are nearly vanished in Raman spectra. This is the case for $v_{\text {as }}(\mathrm{C}-\mathrm{O}-\mathrm{C})$ band around $1240 \mathrm{~cm}^{-1}$, which vibration is associated with direction of main molecular axis. Thus, for Raman based orientation analysis this band is replaced with $\omega\left(\mathrm{CH}_{2}\right)$ around $1284 \mathrm{~cm}^{-1}$ or $v(\mathrm{C}-\mathrm{C})$ around $1037 \mathrm{~cm}^{-1}$.

a)

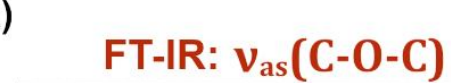

Diffraction limit:

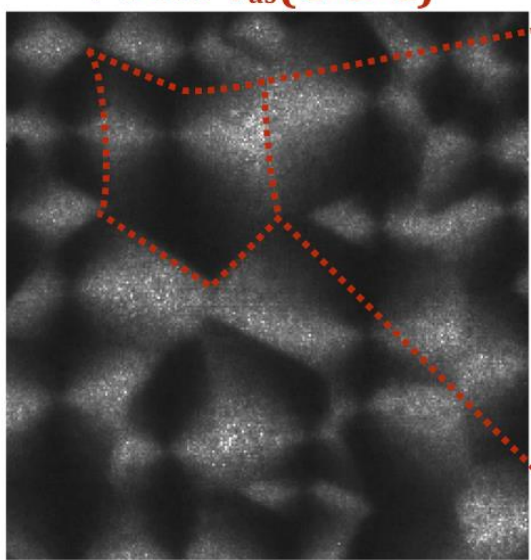
$2.7 \mu \mathrm{m}$ projected pixel size
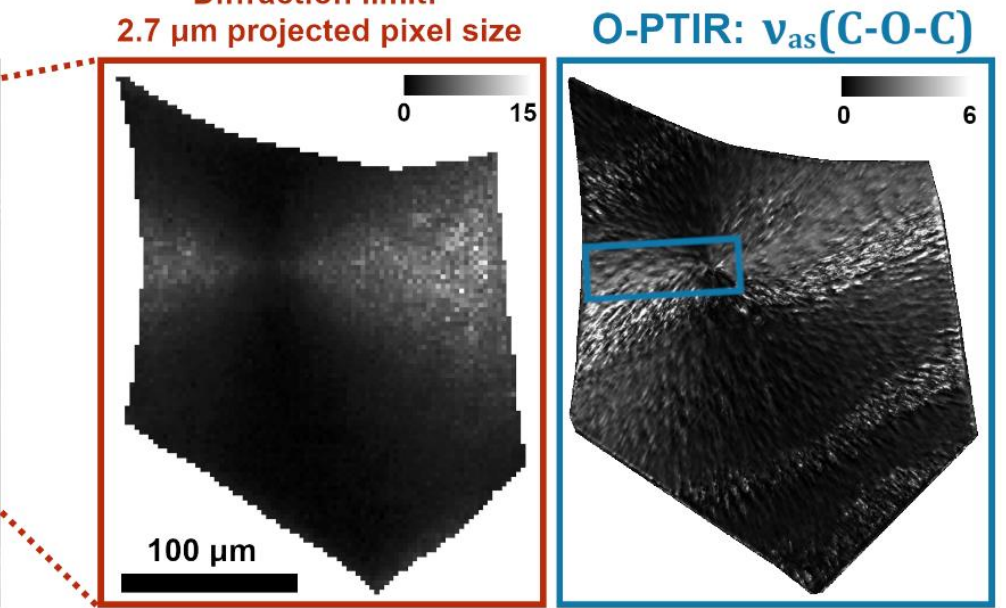

b)

PCL spectra

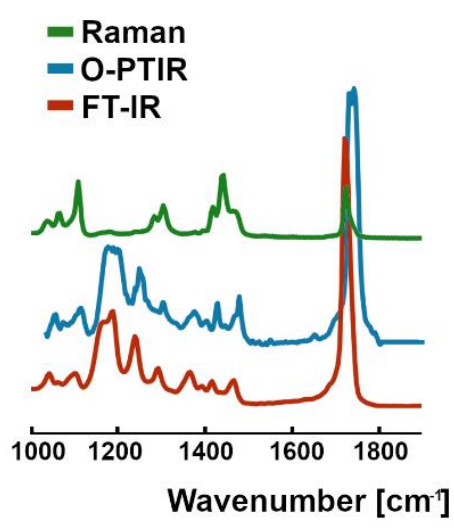

c)

O-PTIR: $v_{\text {as }}(C-0-C)$ Measurement step: $200 \mathrm{~nm}$
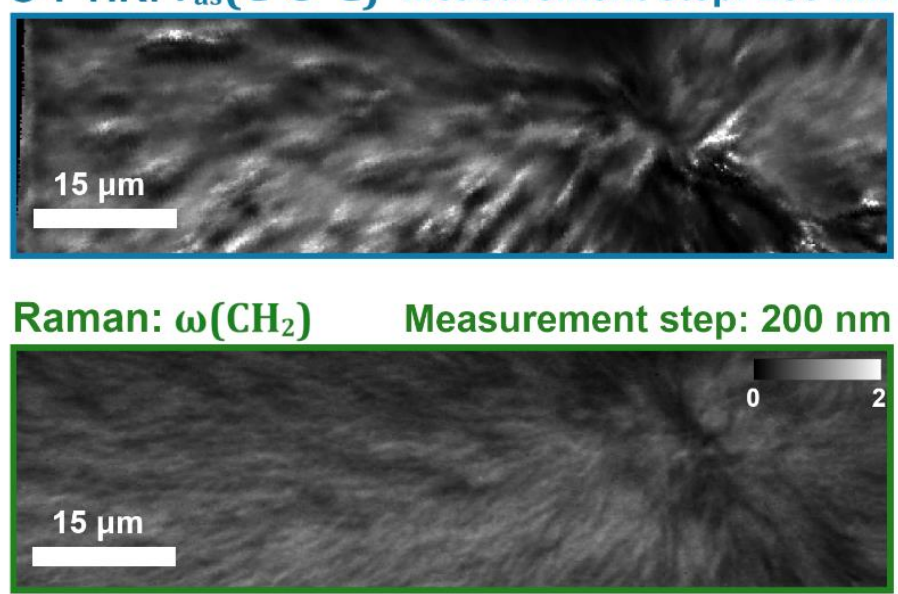
Figure 3. a) Ratio of intensities corresponding to $v_{\text {as }}(\mathrm{C}-\mathrm{O}-\mathrm{C})$ vibrations of PCL sample, collected with two perpendicular polarizations of incident light, using FT-IR and O-PTIR microscopes. Larger polymer film area (covering many spherulites) was acquired for FT-IR, with further attention focused on single spherulite structure. c) Examples of PCL spectra for FT-IR, O-PTIR and Raman techniques (unpolarized), presenting correspondence between FT-IR and O-PTIR and lack of some bands for Raman spectrum. b) Zoom of results shown above (a) for O-PTIR (blue rectangle) with corresponding results (ratio of intensities for two perpendicular polarizations) of the same sample region collected with Raman for $\omega\left(\mathrm{CH}_{2}\right)$ band.

Collected datasets were used to determine orientation of dipole moment and polarizability tensor changes, which can be used to conclude on chemical bonds along with macromolecular orientations. As described in the theoretical section, 3D orientation calculations were done based on pairs of bands intensities, corresponding to transition moments having approximately perpendicular orientations (with respect to each other). Exemplary results are presented in Figure 4 for $v_{\text {as }}(\mathrm{C}-\mathrm{O}-\mathrm{C})$ used as primary vector ( $v(\mathrm{C}-\mathrm{C})$ in case of Raman) and $v(\mathrm{C}=\mathrm{O})$ as secondary vector. Primary vector was always chosen to be approximately parallel to the polymer chain. Unfortunately, it is a challenging task to clearly visualize large 3D datasets, therefore, in some cases only every second point is presented. Moreover, the large pixel density along with acquired data size for O-PTIR ( $1500 \times 1500$ pixels $)$ made it impossible to present whole spherulite at once, so comparison to Raman data is of focus here.

Considering crystalline structure of lamellae in PCL spherulite presented in Figure 2, conclusions could be done regarding theoretical transition moments orientations. With fibrils being organized radially from nucleation centers, direction of main molecular axis (polymer chain) is expected to be perpendicular to fibril axis. Such prediction is strongly confirmed by $3 \mathrm{D}$ orientation results for $\mathrm{v}_{\mathrm{as}}(\mathrm{C}-\mathrm{O}-\mathrm{C})$ and the best visualization is provided by FT-IR and binned O-PTIR shown in the left part of Figure 4a. The purpose of O-PTIR data binning to yield similar spatial detail (pixel size) as FT-IR was to validate and compare results provided by both techniques. Orientation vectors used for data presentation form concentric circles with the center located exactly within the nucleation site area, which confirms that polymer chain is perpendicular with respect to the fibril's axis. Both techniques significantly differ in the optical setup and artifacts coming from scattering and varying sample thickness are also not the same. But regardless of that, the obtained results are consistent with theory and each other. However, the exact orientation of primary transition moment depends on lamellae alignment which can vary from flat-on through twisted to edge-on (Figure 2a). Based on FT-IR and binned O-PTIR orientation of $v_{\text {as }}(\mathrm{C}-\mathrm{O}-\mathrm{C})$ one may conclude that the whole spherulite consists of fibrils formed with edge on lamellas (flat-on lamellas should exhibit orientation perpendicular to the figure plane). Nonetheless, in this case pixel size $(\sim 2.7 \mu \mathrm{m})$ is close to that of lamellae, therefore, averaging of signal coming from this region causes significant loss of observed details. O-PTIR and Raman with relatively small pixel $(200 \mathrm{~nm}$ ) size come to aid, with results for region of spherulite including nucleation site (blue rectangle) presented in the right part of Figure 4a. As expected, comparison of $v_{\text {as }}(\mathrm{C}-\mathrm{O}-\mathrm{C})$ for O-PTIR and $v(\mathrm{C}-\mathrm{C})$ for Raman shows great similarities, with some differences probably caused by different selection rules and contrast mechanism. General structure observed in FT-IR is also preserved in O-PTIR, but there is a striking increase in detail content. Heterogeneity of the sample is clearly visible with wavy structures perhaps corresponding to fibrils shapes. At such magnification, it cannot be stated that spherulite is built exclusively from edge-on lamellas, because both parallel and out of the figure plane vectors are observed in different sample regions.

Interesting results are also observed for secondary transition moment (Figure $4 \mathrm{~b})$. The $v(C=\mathrm{O})$ vibration should be approximately perpendicular to the molecular chain, but it is hard to predict their exact theoretical orientation even if lamellae alignment (edge-on or flat-on) would be determined. Therefore, it is interesting that for FT-IR and binned O-PTIR (left sector of Figure 4b) vectors are consistently 
oriented perpendicularly to the figure plane. There is a slight tilt in O-PTIR results in comparison with FT-IR, but the vectors general trend is steady, and differences could be again caused by the dissimilarities in optical setups. Raman and O-PTIR once more show analogous results, preserving perpendicular trend with respect to primary vector. Although, vectors are no longer consistently perpendicular to the figure plane and heterogenic wavy shapes can be again observed. A detailed relation between primary and secondary vector might be observed in zoomed in areas for O-PTIR and Raman. Combining information about orientation of primary and secondary vectors one may conclude that the investigated spherulite is mostly built from edge-on lamellae. First, for all observed techniques primary vectors are mostly aligned or slightly out of figure plane, which theoretically should be expected for edge-on units. Such theory is supported by perpendicular character (to the figure plane) of secondary vectors. Obviously, there are regions exhibiting those properties to a different extent, but this is expected since crystallization process is very subtle, and its conditions have a major influence on lamellae alignment.

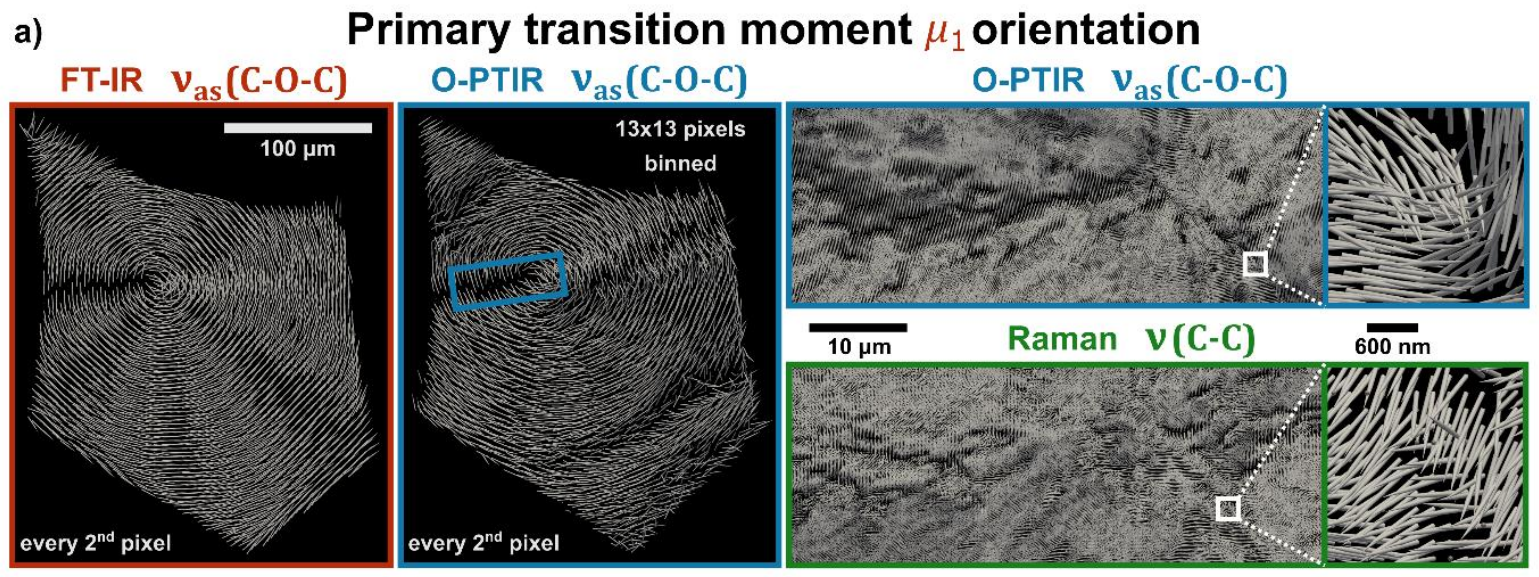

b) Secondary transition moment $\mu_{2}$ orientation
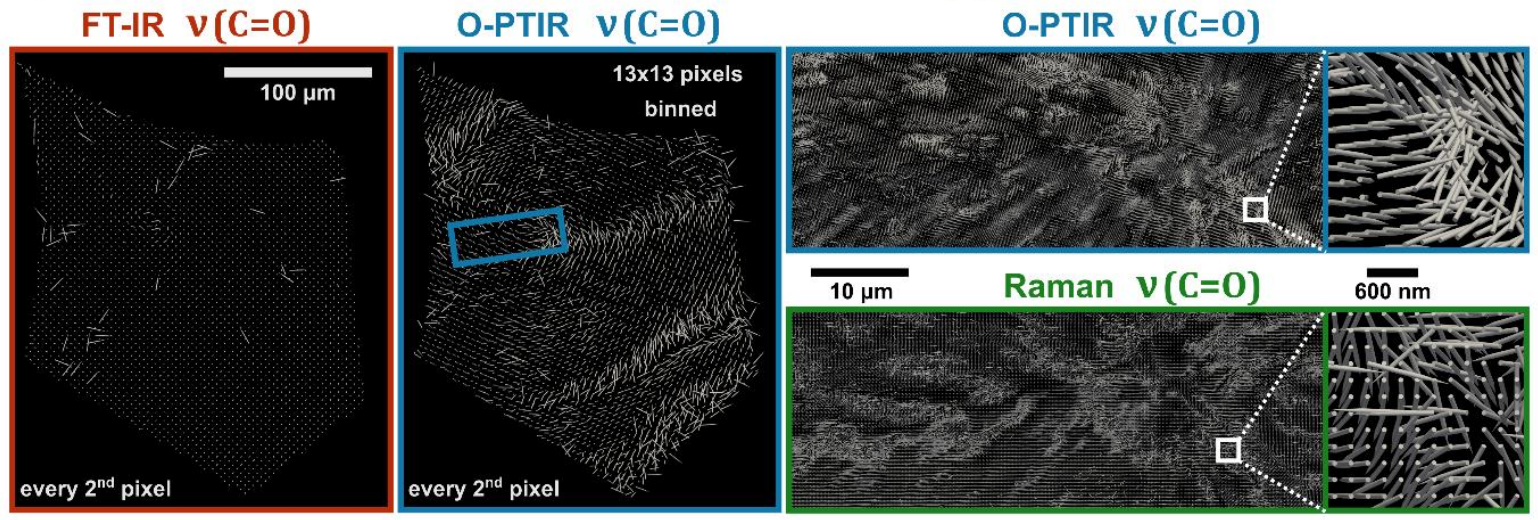

Figure 4. Visualization of primary (b) and secondary (b) transition moment orientation calculated for FT-IR, O-PTIR and Raman. To compare FT-IR and O-PTIR results, O-PTIR data were binned (13x13 pixels) to yield similar pixel size as FT-IR. Orientation results calculated using binned intensities are presented in the middle column. To preserve visualization clarity, results for every second pixels are presented in FT-IR and O-PTIR comparison section. Orientations for a smaller area of spherulite (blue frame covering nucleation center) are presented for O-PTIR (without binning) and Raman in the right column, using their high detail content coming from a diffraction limited image probed with $532 \mathrm{~nm}$ laser. 
Molecular orientation (main polymer axis) and lamellae alignment based on O-PTIR were so far only discussed based on spherulite region corresponding to Raman measurement area to allow for a comparison. Therefore, larger area presenting orientation of $v_{\text {as }}(\mathrm{C}-\mathrm{O}-\mathrm{C})$ is shown in Figure 5a with more structural insight. Once more, the presented region includes nucleation site marked with purple circle, as it is the most characteristic spot in spherulites. But having a look on spherulite's larger area it may be noticed that there are two significantly differentiating domains with zoom-ins marked with white box. Interestingly, after overlaying orientation image presented here with optical microscope image, fibrils are perfectly overlapping with structures formed by vectors in the sample area marked with box on the right side (results shown in Supplementary Materials). However, such structures are not observed in the left zoom where vectors seem to be more parallel to each other. This is regardless of the fact, that fibrils observed in the optical image have the same structure, with no visible differences. This may lead to a conclusion, that there are significant differences within fibrils structures on molecular level, probably associated with growth phases. As explained earlier (Figure 2a), at the early stages of spherulite's formation there is a single fiber, which later becomes surrounded by other radially oriented fibers and branches until full spherulite is formed. As stated before, based on results analyzed so far, this spherulite seems to be mostly built out of edge-on lamellas, which is expected for samples with film thickness larger than $500 \mathrm{~nm}$ [27]. Yet, the observed dissimilarities can arise directly from growth stages.

To have a numerical quantity allowing to visualize and compare some regions of spherulite, a relative in-plane orientation $\psi_{r}$ was defined as difference between $\psi$ angle and direction of fibers. In other words, $\psi_{r}$ describes an in-plane angle relative to the radial axes formed by spherulite's fibrils. Exemplary results for $v_{\text {as }}(\mathrm{C}-\mathrm{O}-\mathrm{C})$ vibration (O-PTIR) are shown in Figure 5b. Presented region directly corresponds to the orientation in Figure 5a. As expected, majority of results exhibit values around $90^{\circ}$ (R1 region), apart from nucleation center which is amorphous. Nevertheless, there are some regions with different orientations $-\mathrm{R} 2$ and R3. Mean values of $\psi_{r}$ for all three regions are presented in Figure $5 \mathrm{c}$, including orientation of other vibrations. Additionally, values for $\theta_{e}=\frac{\pi}{2}-\theta$ angle (elevation) are also presented. A combination of those results allows to analyze relative orientations of specific transition dipole moments. Orientation of $v_{\text {as }}(\mathrm{C}-\mathrm{O}-\mathrm{C})$ is parallel to the PCL polymer main chain axis, whereas $v_{\mathrm{s}}(\mathrm{C}-\mathrm{O}-\mathrm{C})$ should be perpendicular [28]. Here both vectors have similar values of $\psi_{r}$, but $\theta_{e}$ differs around $90^{\circ}$ for all three regions, which confirms perpendicular character of those two. Very similar results are observed for $v_{\text {as }}(\mathrm{C}-\mathrm{O}-\mathrm{C})$ and $v(\mathrm{C}=\mathrm{O})$ pair. The $v(\mathrm{CC}-\mathrm{O})$ vibration is slightly more complex, coming from $\mathrm{C}-\mathrm{O}$ and $\mathrm{C}-\mathrm{C}$ stretching in the crystalline phase [28], but obtained results show that transition dipole moments have direction parallel to the main chain axis, similarly to $v_{\text {as }}(\mathrm{C}-\mathrm{O}-\mathrm{C})$. The same orientation is also observed for $\omega\left(\mathrm{CH}_{2}\right)$. Thus, those three may be considered as vibrations determining main molecular axis orientation. 


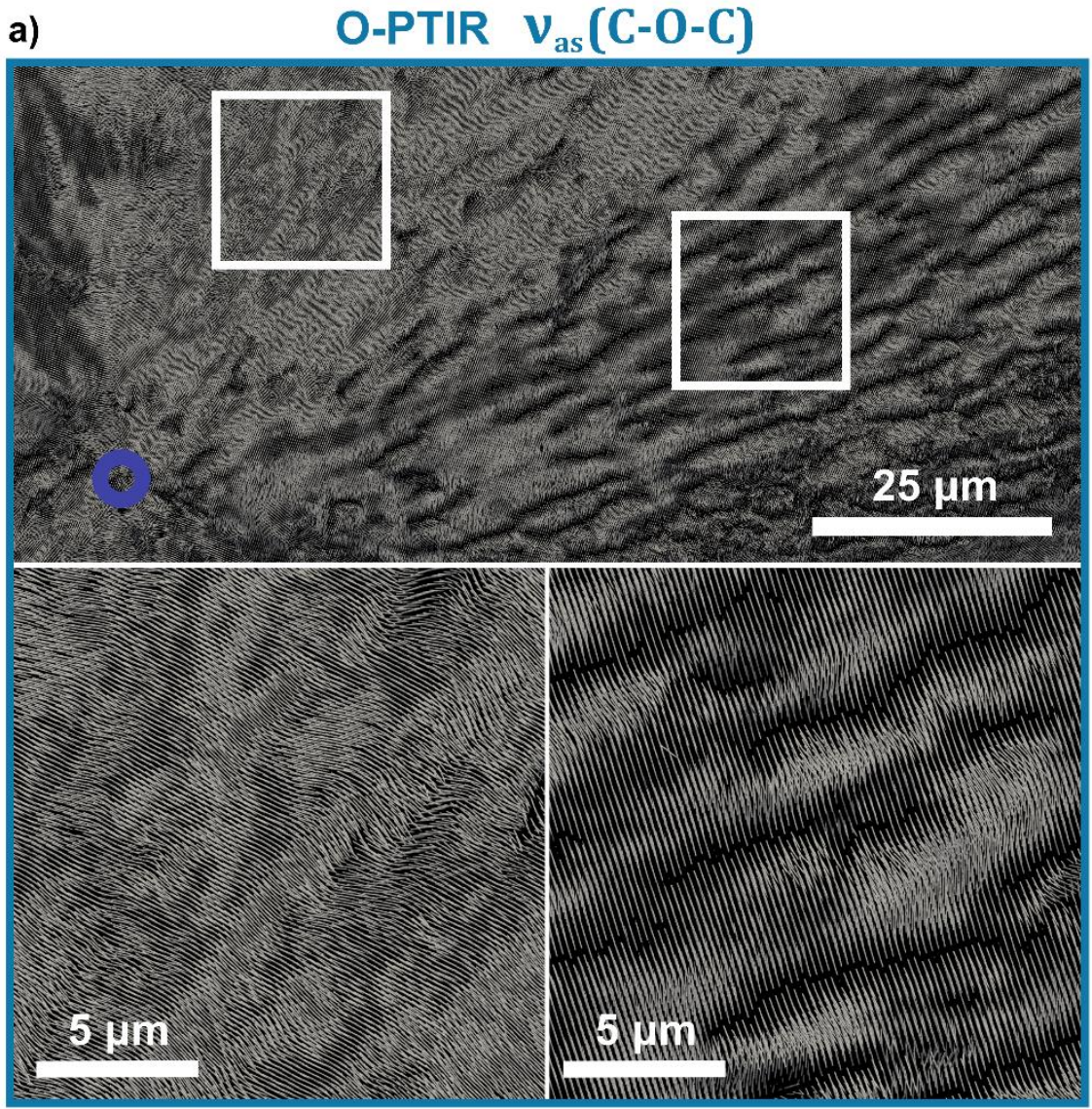

b) In-plane angle relative to radial axes $\psi_{r}$

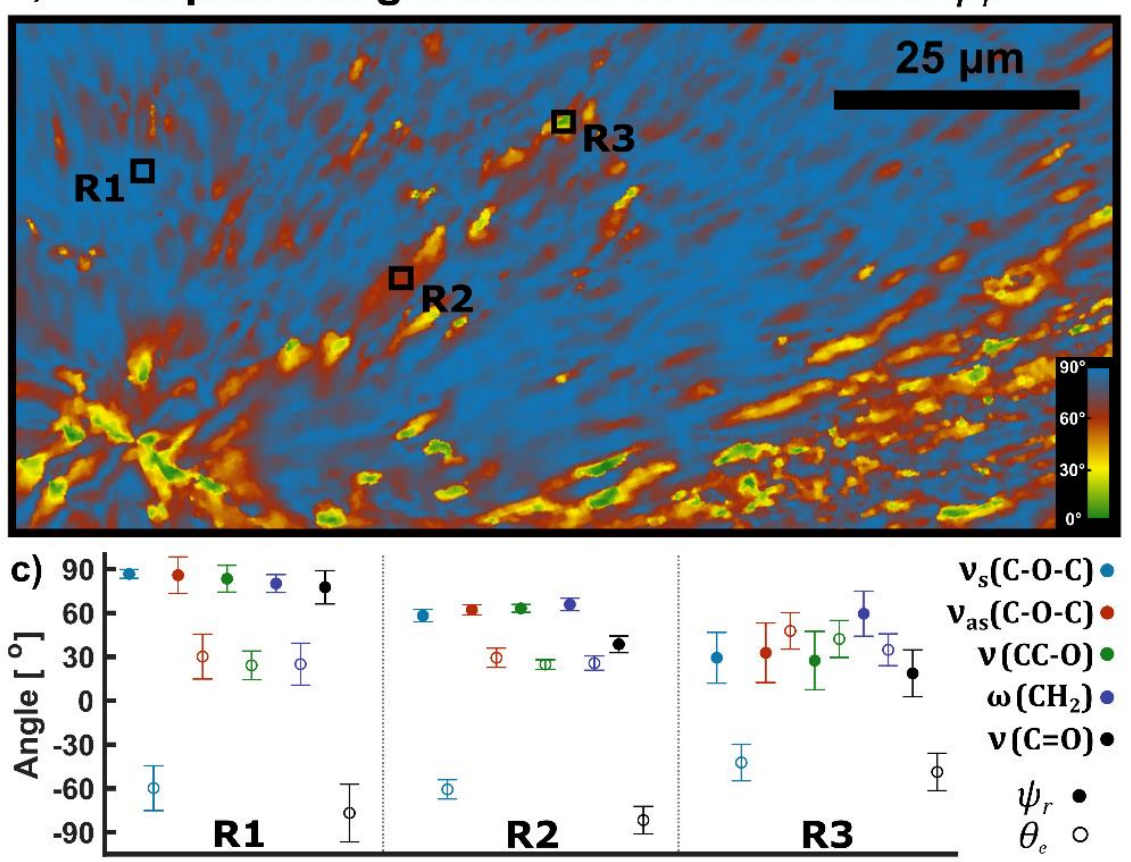

Figure 5. (a) Orientation for $v_{\text {as }}(\mathrm{C}-\mathrm{O}-\mathrm{C})$ dipole moment based on O-PTIR dataset. Zoomed areas are pointing to two clearly distinguishable sample regions, which may be related to areas of different spherulite growth phases. Nucleation site is marked with purple circle. (b) In-plane orientation relative to the radial axes $\psi_{r}$ for $v_{\mathrm{as}}(\mathrm{C}-\mathrm{O}-\mathrm{C})$. (c) Mean values of $\psi_{r}$ and $\theta_{e}$ for three regions (R1, R2 and R3) for different dipole moments along with standard deviations within that region. 


\section{Conclusions}

We successfully demonstrated for the first time the four-polarization method coupled to concurrent vibration analysis on a spherulite polymer sample. We show that this framework gives consistent results across there imaging modalities, each at their diffraction limit. It opens applications for spatially resolved orientation studies not limited to ultra-thin samples, which can be measured in a nondestructive, label-free manner. This new method is expected to impact primarily material and biological sciences as information previously unattainable can be extracted without a complicated experimental setup.

\section{Acknowledgements}

This research was supported the National Science Centre, Poland (Grant No. 2018/31/D/ST4/01833). Part of this research was performed using equipment purchased in the frame of the project co-funded by the Malopolska Regional Operational Program Measure 5.1 Krakow Metropolitan Area as an important hub of the European Research Area for 2007-2013, project no. MRPO.05.01.00-12-013/15. O-PTIR measurements were done at the SMIS beamline of the SOLEIL synchrotron facility. FT-IR measurements were in part done at the SOLAIR beamline of the SOLARIS synchrotron facility. The authors declare no conflicts of interest

\section{References}

[1] Y. Xu et al., "Nanostructured polymer films with metal-like thermal conductivity," Nature Communications, vol. 10, no. 1, Dec. 2019, doi: 10.1038/s41467-019-09697-7.

[2] W. Zhang, P. Feng, J. Chen, Z. Sun, and B. Zhao, "Electrically conductive hydrogels for flexible energy storage systems," Progress in Polymer Science, vol. 88, Jan. 2019, doi: 10.1016/j.progpolymsci.2018.09.001.

[3] J. Kang et al., "Tough and Water- Insensitive Self- Healing Elastomer for Robust Electronic Skin," Advanced Materials, vol. 30, no. 13, Mar. 2018, doi: 10.1002/adma.201706846.

[4] K. Zhang et al., "Advanced smart biomaterials and constructs for hard tissue engineering and regeneration," Bone Research, vol. 6, no. 1, Dec. 2018, doi: 10.1038/s41413-018-0032-9.

[5] E. Hecht, Optics (4th Edition). Addison-Wesley, 2012.

[6] T. P. Wrobel and R. Bhargava, "Infrared Spectroscopic Imaging Advances as an Analytical Technology for Biomedical Sciences," Analytical Chemistry, vol. 90, no. 3, pp. 1444-1463, Feb. 2018, doi: 10.1021/acs.analchem.7b05330.

[7] D. Zhang, C. Li, C. Zhang, M. N. Slipchenko, G. Eakins, and J.-X. Cheng, "Depth-resolved midinfrared photothermal imaging of living cells and organisms with submicrometer spatial resolution," Science Advances, vol. 2, no. 9, Sep. 2016, doi: 10.1126/sciadv.1600521.

[8] Y. Su et al., "Steam disinfection releases micro(nano)plastics from silicone-rubber baby teats as examined by optical photothermal infrared microspectroscopy," Nature Nanotechnology, Nov. 2021, doi: 10.1038/s41565-021-00998-x.

[9] S. F. S. P. Looijmans et al., "Polarization modulated infrared spectroscopy: A pragmatic tool for polymer science and engineering," POLYMER CRYSTALLIZATION, vol. 3, no. 6, Dec. 2020, doi: $10.1002 /$ pcr2.10138. 
[10] K. Bocz et al., "Non-destructive characterisation of all-polypropylene composites using small angle X-ray scattering and polarized Raman spectroscopy," Composites Part A: Applied Science and Manufacturing, vol. 114, Nov. 2018, doi: 10.1016/j.compositesa.2018.08.020.

[11] Y. Hikima, J. Morikawa, and T. Hashimoto, "FT-IR Image Processing Algorithms for In-Plane Orientation Function and Azimuth Angle of Uniaxially Drawn Polyethylene Composite Film," Macromolecules, vol. 44, no. 10, pp. 3950-3957, May 2011, doi: 10.1021/ma2003129.

[12] Y. Hikima, J. Morikawa, and T. Hashimoto, "Imaging of Two-Dimensional Distribution of Molecular Orientation in Poly(ethylene oxide) Spherulite Using IR Spectrum and Birefringence," Macromolecules, vol. 45, no. 20, pp. 8356-8362, Oct. 2012, doi: 10.1021/ma3010372.

[13] Y. Hikima, J. Morikawa, and T. Hashimoto, "Wavenumber dependence of FT-IR image of molecular orientation in banded spherulites of poly(3-hydroxybutyrate) and poly(l-lactic acid)," Macromolecules, vol. 46, no. 4, pp. 1582-1590, 2013, doi: 10.1021/ma302560q.

[14] M. Ryu et al., "Orientational Mapping Augmented Sub-Wavelength Hyper-Spectral Imaging of Silk,” Scientific Reports, vol. 7, no. 1, Dec. 2017, doi: 10.1038/s41598-017-07502-3.

[15] T. P. Wrobel, P. Mukherjee, and R. Bhargava, "Rapid visualization of macromolecular orientation by discrete frequency mid-infrared spectroscopic imaging," Analyst, vol. 142, no. 1, 2017, doi: 10.1039/c6an01086e.

[16] P. Koziol, D. Liberda, W. M. Kwiatek, and T. P. Wrobel, "Macromolecular Orientation in Biological Tissues Using a Four-Polarization Method in FT-IR Imaging," Analytical Chemistry, vol. 92, no. 19, Oct. 2020, doi: 10.1021/acs.analchem.0c02591.

[17] P. Koziol et al., "Comparison of spectral and spatial denoising techniques in the context of High Definition FT-IR imaging hyperspectral data," Scientific Reports, vol. 8, no. 1, pp. 1-11, 2018, doi: 10.1038/s41598-018-32713-7.

[18] M. K. M. K. Raczkowska et al., "Influence of denoising on classification results in the context of hyperspectral data : High Definition FT-IR imaging," Analytica Chimica Acta, vol. 1085, pp. 39-47, 2019, doi: 10.1016/j.aca.2019.07.045.

[19] P. R. Griffiths and J. A. de Haseth, Fourier Transform Infrared Spectrometry. Hoboken, NJ, USA: John Wiley \& Sons, Inc., 2007. doi: 10.1002/047010631X.

[20] “ATKINS' PHYSICAL CHEMISTRY."

[21] Y. J. Lee, "Concurrent polarization IR analysis to determine the $3 \mathrm{D}$ angles and the order parameter for molecular orientation imaging," Optics Express, vol. 26, no. 19, p. 24577, Sep. 2018, doi: 10.1364/OE.26.024577.

[22] Y. Hikima, J. Morikawa, and T. Hashimoto, "Wavenumber Dependence of FT-IR Image of Molecular Orientation in Banded Spherulites of Poly(3-hydroxybutyrate) and Poly(L-lactic acid)," Macromolecules, vol. 46, no. 4, Feb. 2013, doi: 10.1021/ma302560q.

[23] Y. J. Lee, "Concurrent polarization IR analysis to determine the 3D angles and the order parameter for molecular orientation imaging," Optics Express, vol. 26, no. 19, p. 24577, 2018, doi: 10.1364/oe.26.024577. 
[24] R. Androsch, M. L. di Lorenzo, C. Schick, and B. Wunderlich, "Mesophases in polyethylene, polypropylene, and poly(1-butene)," Polymer, vol. 51, no. 21, pp. 4639-4662, Oct. 2010, doi: 10.1016/j.polymer.2010.07.033.

[25] P. Nguyen-Tri, P. Ghassemi, P. Carriere, S. Nanda, A. A. Assadi, and D. D. Nguyen, "Recent Applications of Advanced Atomic Force Microscopy in Polymer Science: A Review," Polymers, vol. 12, no. 5, p. 1142, May 2020, doi: 10.3390/polym12051142.

[26] J. Xu, H. Ye, S. Zhang, and B. Guo, "Organization of twisting lamellar crystals in birefringent banded polymer spherulites: A mini-review,” Crystals, vol. 7, no. 8. MDPI AG, Aug. 04, 2017. doi: $10.3390 /$ cryst7080241.

[27] B. Crist and J. M. Schultz, "Polymer spherulites: A critical review," Progress in Polymer Science, vol. 56, pp. 1-63, 2016, doi: 10.1016/j.progpolymsci.2015.11.006.

[28] T. Elzein, M. Nasser-Eddine, C. Delaite, S. Bistac, and P. Dumas, "FTIR study of polycaprolactone chain organization at interfaces," Journal of Colloid and Interface Science, vol. 273, no. 2, pp. 381-387, 2004, doi: 10.1016/j.jcis.2004.02.001. 